\title{
DETECÇÃO E CARACTERIZAÇÃO DE ENTEROPARASITOS EM ANIMAIS SILVESTRES DO ESTADO DA BAHIA
}

\author{
Ellen Monteiro Ribeiro Santos ${ }^{1}$; Aristeu Vieira da Silva ${ }^{2}$; Ilmara Simony Freitas \\ Santana $^{3}$ e Priscylla Marcelly Vila Nova ${ }^{4}$ \\ 1. Bolsista PIBIC/CNPq, Graduando em Ciências Biológicas, Universidade Estadual de Feira de Santana, e-mail: \\ ellenmonteiro28@hotmail.com \\ 2. Orientador, Grupo de Pesquisa em Zoonoses e Saúde Pública, Departamento de Ciências Biológicas, Universidade \\ Estadual de Feira de Santana, e-mail: aristeuvsilva@uefs.br; bolsista de produtividade CNPq \\ 3. Mestranda do Programa de Pós-Graduação em Ecologia e Evolução, Departamento de Ciências Biológicas, Universidade \\ Estadual de Feira de Santana, e-mail: ylmara_santana@ hotmail.com; bolsista CAPES \\ 4. Participante do projeto, Departamento de Ciências Biológicas, Universidade Estadual de Feira de Santana, e-mail: \\ priscylla.marcelly@hotmail.com ; bolsista PROBIC-UEFS
}

PALAVRAS-CHAVE: Zoonoses; Enteroparasitos; Animais Silvestres.

\section{INTRODUÇÃO}

O desenvolvimento do capitalismo desencadeou rápidos processos de urbanização, afetando assim de forma drástica o meio ambiente ocasionando uma perda progressiva da biodiversidade em consequência da fragmentação do habitat e do desequilíbrio ecológico. Essas alterações são responsáveis pela migração de algumas espécies selvagens para o perímetro periurbano (MACHENSTEDT, 2015), favorecendo as interações da fauna silvestre com animais domésticos e humanos, aumentando assim a probabilidade de transmissão e disseminação de doenças zoonóticas.

A prevalência de parasitos intestinais pode variar de acordo com fatores demográficos, geográficos, climáticos, comportamentais e populacionais do hospedeiro. A natureza da infecção nos animais, possivelmente está interligada ao tipo de nicho ecológico dentro do seu habitat natural, possibilitando assim atuarem como hospedeiros intermediários, paratênicos ou definitivos. (RUAS, 2008)

$\mathrm{O}$ estudo das zoonozes permite avaliar o potencial de animais selvagens como propagadores de doenças infecciosas em humanos e constatar as alterações da dinâmica dentro das populações silvestres em decorrência da prevalência dos parasitos (THOMPSON,2009).

\section{MATERIAL E MÉTODOS OU METODOLOGIA (ou equivalente)}

O trato gastro-intestinal de cada animal foi retirado e os instrumentos utilizados para cada animal foram limpos com uma solução de hipoclorito de sódio (2,5\%) para evitar contaminação cruzada entre as amostras. As amostras de conteúdo gastro-intestinal foram submetidas ao método de Willis e Faust (SLOSS et al.,1999), consistindo na homogeneização das fezes em água destilada, e ciclos de centrifugação e troca do sobrenadante até clarificação da suspensão, ressuspendendo-se o sedimento final em soluções hipersaturadas de cloreto de sódio (Willis) e de sulfato de zinco (Faust), centrifugando a solução novamente, permitindo a separação das estruturas leves, como ovos de helmintos, cistos e oocistos de protozoários na película superficial, que foi colhida com alça de platina, depositada em lâmina de microscopia, adicionado lugol e examinadas sobre microscopia óptica para presença de enteroparasitos identificados segundo Foreyt (2001).

Foram inclusas no projeto amostras fixadas em álcool, da coleção de mastofauna do Museu de Zoologia da Universidade Estadual de Feira de Santana, de forma que a maioria das 
amostras tiveram seu conteúdo estomacal e intestinal (quando presente) removidos e reservados em tubos Falcon de $15 \mathrm{~mL}$ para realização dos exames coprológicos, e as demais amostras por não apresentarem conteúdo (ou apresentarem quantidades abaixo de 1,5 mL) estomacal e intestinal, foram trituradas em mixer com álcool em quantidade equivalente ao seu peso multiplicado por quatro.

\section{RESULTADOS E/OU DISCUSSÃO (ou Análise e discussão dos resultados)}

Um total de 24 amostras foram analisadas, nas quais estavam representados marsupiais das espécies Didelphis aurita (Gambá-de-orelha-preta) e Metachirus nudicaudatus (Cuícacauda-de-rato), roedores das espécies Akodon cursor (Rato-do-chão), Nectomys squamipes (Rato-d'água) e Trinomys cf. T. setosus (Rato-de-espinho), quirópteros das espécies Artibeus lituratus, cf. Myotis, Rhynchonycteris sp, Desmodus rotundus (Morcego-vampiro) e Platyrrhinus lineatus, e canídeos da espécie Cerdocyon thous (Cachorro-do-mato).

Do total de amostras fecais, duas amostras apresentaram resultados positivos para a presença de ovos de helmintos, as demais amostras, apesar de apresentarem negatividade nos exames, foram encontrados parasitos em estágio adulto em cinco amostras: dois roedores da espécie Nectomys squamipes (Rato-d'água), um roedor do gênero Cerradomys sp. e dois marsupiais da espécie Metachirus nudicaudatus (Cuíca-cauda-de-rato), indicando assim a presença de enteroparasitoses desses animais (Figura 1). Cabe ressaltar a baixa sensibilidade dos exames parasitológicos das fezes nestes casos, uma vez que a presença de helmintos adultos condicionavam o parasitismo. Além disso, o método de Faust foi bem menos sensível que o método de Willis, detectando apenas uma amostra positiva para Isospora spp. Em um C. thous, enquanto que o método de Willis possibilitou o encontro de nematódeos e cestódeos nos dois C. thous examinados.

Os parasitos adultos puderam ser identificados apenas ao táxon Filo (Figura 1), pois a conservação em álcool não permitiu que se aplicasse técnicas de diafanização e coloração dos espécimes, para que se verificasse estruturas morfológicas que permitissem a identificação das famílias, gêneros ou espécies envolvidas. Desta forma, os vermes adultos foram mantidos congelados em álcool absoluto, para posterior extração de DNA e aplicação de técnicas moleculares para se determinar as espécies envolvidas. Dos 27 animais examinados para a presença de parasitos adultos, cinco $(18,5 \%)$ foram positivos, sendo encontrados dois parasitos em um Nectomys squamipes, dez em um Metachirus nudicaudatus, doze em um N. squamipes, 25 em um Cerradomys sp e 55 em um M. nudicaudatus.

Figura 1. Microfotografia (aumento de 20x) de Nematoda (a) e Platelmynthe (b, c) encontrados em Nectomys squamipes (figura a) e Cerradomys sp (figura b e c), da Colecão de Mamíferos do Museu de Zoologia da Universidade Estadual de Feira de Santana. Feira de Santana, 2017.

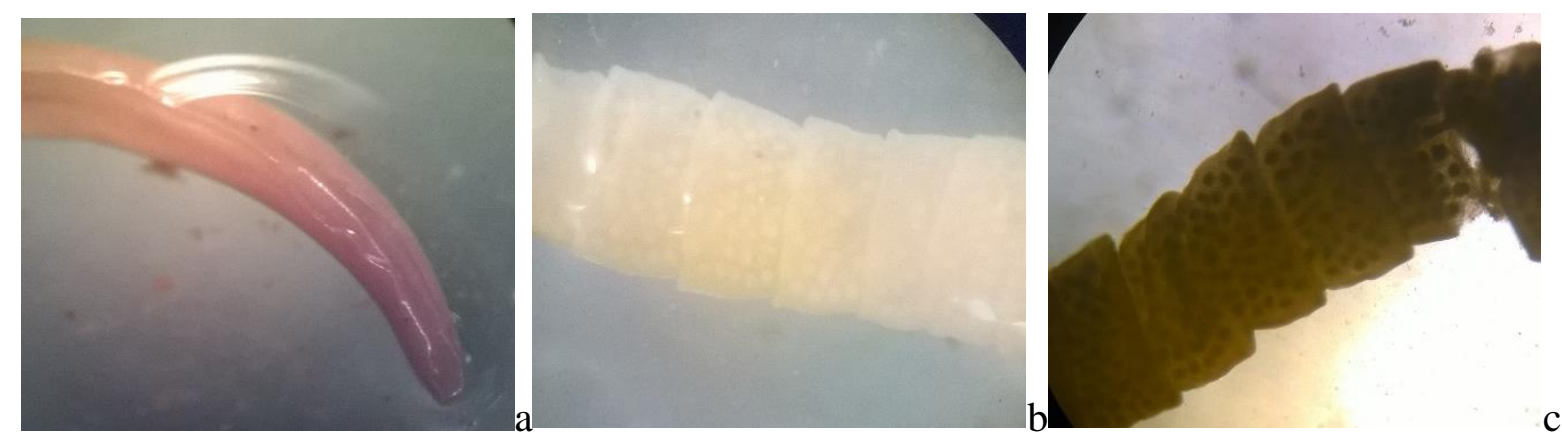


Gomes e Vicente (1984) determinaram a presença de enteroparasitos em 71 Nectomys squamipes necropsiados em Sumidouro (RJ), tendo encontrado parasitos em $49(59,15 \%)$ dos animais examinados, encontrando Trematoda (Pseudochinostomum caballeroi), Cestoda (Raillietina sp) e Nematoda (Hassalstrongylus épsilon, Syphacia venteli, Aspidodera raillieti, Physaloptera bispiculata e Litomosoides carinii). Quanto a Cerradomys, o trabalho de Barreto et al (2015) não registra a presença de helmintos, mas apenas de Isospora em um $(11,1 \%)$ de nove roedores examinados. Em Metachirus já foram descritos o parasitismo por Aspidodera subulata (ARAÚJO, 2011).

As amostra positivas foram de dois canídeos Cerdocyon thous, que a partir dos métodos de Willis e Faust (SLOSS et al. ; 1999) indicaram a presença de ovos dos seguintes gêneros : Thichuris spp., Capillaria spp., Moniezia spp., Ascaridia spp., Tricostrongyllus spp., Isospora spp., Toxocara spp. e ancilostomídeos (Figura 2). Gomes et al (2015) registram a ocorrência dos seguintes parasitos em três $C$. thous recolhidos em rodovias de São Paulo: Ancylostoma buckleyi, Pterygodermatites pluripectinata e Ascaridia galli. Vieira (2011) relatam o encontro de Dypillidium caninum em C. thous de Juiz de Fora (MG). Lima (2009), trabalhando com 59 C. thous recolhidos de atropelamentos em rodovias da Paraiba, relatam a presença de 16 espécies de helmintos, sendo dois trematódeos, um cestódeos, um acantocéfalo e 12 nematódeos, confirmando a maior diversidade de Nematelmintos nesta espécie de carnívoro.

Figura 2. Micrografias (aumento de 400x) de ovos de helmintos encontrados ao exame das fezes de Cerdocyon thous encontrados atropelados no município de Cruz das Almas. Universidade Estadual de Feira de Santana. Feira de Santana, 2017.

a) Trichuris spp.; b) Ascaridia spp.; c) Capillaria spp.; d) Moniezia spp.; e) Toxocara spp.; f) Trychostrongylus spp.
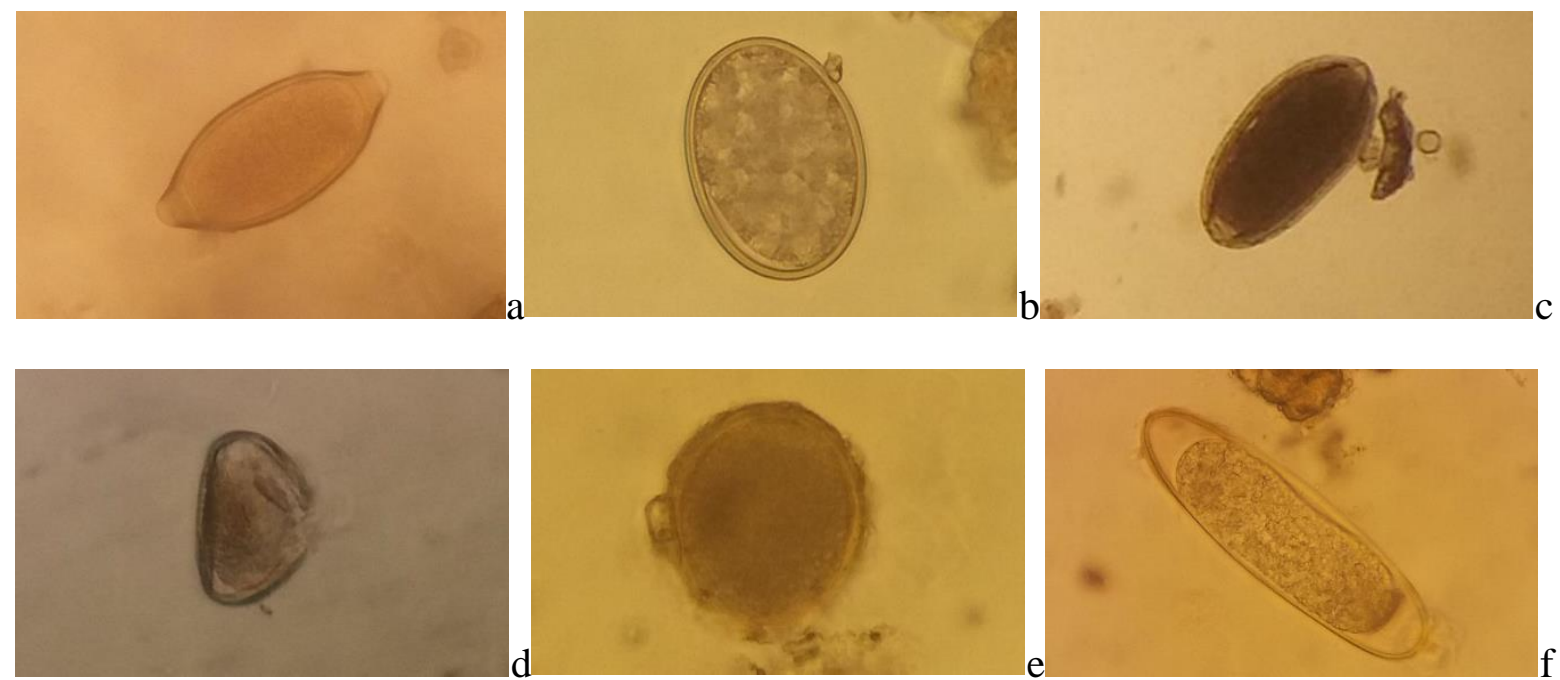

O baixo número de positivos está provavelmente relacionado a conservação das amostras devido ao longo período de tempo que as mesmas permaneceram preservadas em álcool.

\section{CONSIDERAÇÕES FINAIS}

De acordo com os resultados é possível inferir que a conservação das amostras e o estado das mesmas influencia diretamente na avaliação da abundância e frequência de parasitos, sendo mais viável para esse tipo de estudo e metodologia a utilização de amostras fecais frescas. A partir das amostras frescas foi possível avaliar qualitativamente a presença de enteroparasitoses 
em canídeos possivelmente relacionadas com a migração desses indivíduos para perímetro periurbano. Apesar da maior incidência de negativos o trabalho desenvolvido é de grande importância para determinar a prevalência de parasitos em animais silvestres, permitindo a compreensão dos mesmos como agentes zoonoticos.

\section{REFERÊNCIAS}

MACHENSTEDT, Ute; JENKINS, David; ROMIG, Thomas. The role of wildlife in the transmission of parasitic zoonoses in peri-urban and urban áreas. In: International Journal for Parasitology: Parasites and Wildlife 4, p.71-79,2015. Disponivel em: < http://ac.els-cdn.com/S2213224415000085/1-s2.0-S2213224415000085main.pdf? tid=685f635a-7328-11e7-804c$\underline{00000 \mathrm{aab} 0 \mathrm{f} 27 \& \text { acdnat }=1501200484 \_51 \mathrm{c} 581608 \mathrm{f} 0389 \mathrm{acd} 0 \mathrm{edea} 210 \mathrm{e} 2 \mathrm{f} 2690}>$ Acesso em : 27 de julho de 2017

THOMPSON, Rc Andrew; KUTZ, Susan J.; SMITH, Andrew. Parasite Zoonoses and Wildlife: Emerging Issues. In: International Journal Of Environmental Research And Public Health, S.1, v. 6, n. 2, p.678-693, 13 fev. 2009. Semestral. Disponível em: 〈http://www.mdpi.com/1660-4601/6/2/678/htm〉. Acesso em: 28 jul. 2017

RUAS, Jerônimo L. et al. HELMINTOS DO CACHORRO DO CAMPO, Pseudalopex gymnocercus (FISCHER, 1814) E DO CACHORRO DO MATO, Cerdocyon thous (LINNAEUS, 1766) NO SUL DO ESTADO DO RIO GRANDE DO SUL, BRASIL. Revista Brasileira de Parasitologia Veterinária, Rio Grande do Sul, v. 2, n. 17 , p.87-92, 2008. Disponível em: <http://www.scielo.br/pdf/rbpv/v17n2/05.pdf>. Acesso em: 01 ago. 2017.

GOMES, Delir Corrêa; VICENTE, J.Julio. Helmintos Parasitos de Nectomys Squamipes (Brants) do Município de Sumidouro,RJ. Instituto Oswaldo Cruz, Rio de Janeiro, v. 79, n. 1, p.67-73, mar. 1984

Congresso de Ecologia do Brasil, 12., 2015, S.1. DIVERSIDADE BIOLÓGICA DE PROTOZOÁRIOS COCCÍDEOS EM PEQUENOS MAMÍFEROS NO PANTANAL DE MATO GROSSO DO SUL. Campo Grande-MS: Sociedade de Ecologia do Brasil, 2015. 2 p.

ARAUJO, Laudemir Roberto Ferreira. "DESCRIÇÃO TAXONÔMICA DE Cruzia sp. nov. e Aspidodera sp. nov. (NEMATODA, ASCARIDIDA), PARASITAS DE INTESTINO GROSSO DE Philander opossum LINNAEUS, 1758, MARSUPIAL DE CARAJÁS-PARÁ, BRASIL”. 2011. 86 f. Dissertação (Mestrado) Curso de Ciências Biológicas, Universidade Federal do Pará Instituto de Ciências Biológicas Programa de Pósgraduação Biologia de Agentes Infecciosos e Parasitários, Belém-PA, 2011

GOMES, Ana Paula Nascimento et al. New records of three species of nematodes in Cerdocyon thous from the Brazilian Pantanal wetlands. Revista Brasileira de Parasitologia Veterinária, [s.1.], v. 24, n. 3, p.324-330, set. 2015. FapUNIFESP (SciELO). http://dx.doi.org/10.1590/s1984-29612015061. Disponível em: $<$ http://www.scielo.br/scielo.php?script=sci_arttext\&pid=S1984-29612015000300324\&lng=en\&tlng=en $\quad>$. Acesso em: 04 ago. 2017.

VIEIRA, Fabiano Matos. Helmintos parasitos de mamíferos carnívoros silvestres no Município de Juiz de Fora, Zona da Mata do Estado de Minas Gerais, Brasil. 2011. 86 f. Tese (Doutorado) - Curso de Ciências Biológicas, Universidade Federal Rural do Rio de Janeiro, Seropédica, Rj, 2011. Disponível em: <http://www.ufrrj.br/posgrad/cpgba/teses/Tese Doutorado Fabiano pdf PGBA.pdf>. Acesso em: 01 ago. 2017.

LIMA, Roberto César Araújo de. HELMINTOS GASTRINTESTINAIS DE Cerdocyon thous (LINNAEUS, 1766) SMITH, 1839 PROVENIENTES DA ÁREA DE CAATINGA DO ESTADO DA PARAíbA, BRASIL. 2007. 61 f. Dissertação (Mestrado) - Curso de Medicina Veterinária, Universidade Estadual Paulista Faculdade de Ciências Agrárias e Veterinárias Câmpus de Jaboticabal, Jaboticabal- Sp, 2009 